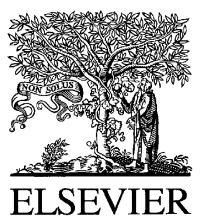

\title{
Multiple prey traits, multiple predators: keys to understanding complex community dynamics
}

\author{
Thomas J. DeWitt*, R. Brian Langerhans \\ Department of Wildlife and Fisheries Sciences, Texas A\&M University, 2258 TAMU, College Station, TX 77843-2258, USA
}

Received 26 September 2002; accepted 20 November 2002

\begin{abstract}
Natural communities can be complex. Such complexity makes it difficult to discern the mechanisms generating community structure. In this paper we review concepts and issues related to linking functional and community studies while also including greater complexity into the experimental realm. These principles are primarily illustrated with case studies involving predation ecology in a freshwater snail-fish-crayfish model system. The system illustrates how predator impacts on prey are mediated by multiple prey traits, correlations between traits, functional trade-offs in predator defence, interactions between predators, and interactions with other community members. We argue for a pluralistic approach to investigating mechanisms of community structure; that is, an approach that integrates many subdisciplines of ecology and evolution. We discuss four main areas that when used together yield important insights on community structure. First, selection gradient analyses formally link functional and community ecology. This formalisation is shown to help identify targets of selection, estimate environment-specific mortality rates, and identify agents of selection in complex communities. Second, we encourage increased focus on emergent community properties (results not predicted based on pairwise species interactions). Third, we emphasise that a community, rather than a web of species interactions, may more profitably be viewed as a network of trait interactions. This trait-centred view makes clear how indirect community effects arise between species that do not interact physically. This perspective also leads to our fourth topic, the integration of phenotypes. Just as populations evolve co-adapted suites of traits, so too should individuals embody integrated trait correlations, termed 'trait integration', rather than randomly assembled collections of phenotypes. All the perspectives mentioned above suggest that investigations should focus on multiple traits and multiple environments simultaneously, rather than singular, atomised components of complex systems.
\end{abstract}

(C) 2002 Elsevier Science B.V. All rights reserved.

Keywords: Selection gradient analysis; Trait integration; Emergent impacts; Indirect effects; Co-adaptation; Physa; Food webs

\section{Introduction}

A multitude of interactions contribute to community structure (reviewed in Polis and Winemiller,

* Corresponding author.

E-mail address: tdewitt@tamu.edu (T.J. DeWitt).
1996). Such complexity makes it difficult to discern the mechanisms generating pattern in natural communities. Complexity also implies that no single approach to the study of community structure is likely to succeed. In particular, we would like to begin by reiterating a point made by many naturalists previously, that processes underlying community 
structure are both ecological and evolutionary in nature (Losos, 1990, 1996; Winemiller, 1991; McPeek, 1996; Miller and Travis, 1996; Webb, 2000). Although it is still unclear at this point to what degree deterministic as opposed to stochastic processes contribute to community patterns (e.g. Gotelli and Graves, 1996; Bell, 2001; Hubbell, 2001), for purposes here we will address nonrandom processes. Our thesis is that to understand this natural complexity, one must integrate many subdisciplines of ecology and evolution. That is, a pluralistic approach is required to continue increasing our understanding of natural communities. To shed light on this approach, we first discuss combining ecological and evolutionary perspectives, then follow with some specific aspects of ecological integration centred around multiple traits and multiple agents of selection.

Reasons for integrating aspects of ecology and evolution in community ecology are numerous. First, community ecologists will benefit greatly from borrowing a tool from evolutionary biology: selection gradient analysis. Selection gradient analysis, as we argue, emerges as a natural extension of functional ecology which parameterises both ecological and evolutionary effects simultaneously. When applied as we present in this paper, this procedure generates parameters describing impacts of one or a set of species on the abundance and trait distributions of a focal species. So one formula can describe both the relative mortality suffered by a species and the magnitude and direction of selection acting on specific traits. This wedding of ecology and evolution provides the type of synthesis ecologists have called for in recent years (e.g. see articles in Ecology 77:5).

Second, due to the complex nature of interactions within communities, emergent properties arise producing patterns not predicted based on pairwise interactions. For example, knowing the impact of predator A on a prey species, and knowing predator B's impact, one may not simply sum these interactions and accurately predict responses in the presence of both predators (Soluk, 1993; Sih et al., 1998).

Third, to fully understand how community patterns emerge from a complex network of interacting species and abiotic processes, we must simultane- ously address multiple components of a community and examine all relevant interactions. Constituent species within communities are often linked through both direct and indirect interactions (Kerfoot, 1987; Wootton, 1994). Direct effects result from physical interaction between two species, while indirect effects require the presence of intermediary species or a set of physical or chemical variables. For example, a common indirect effect is exploitative competition, where one species impacts the density of another species through consumption of shared prey (Connell, 1983; Shoener, 1983; Goldberg and Barton, 1992; Wootton, 1994; Grover, 1997). There are two major types of indirect effects (Wootton, 1993). The previous example represents a densitymediated indirect effect; however, indirect effects also arise when one species influences how two other species interact (i.e. trait-mediated indirect effect, Abrams et al., 1996). Trait-mediated indirect effects commonly involve predator-induced behavioural shifts in a prey, which have cascading effects on prey resources (e.g. Lima and Dill, 1990; Turner and Mittelbach, 1990; Huang and Sih, 1991; Werner, 1992; Schmitz et al., 1997; Ripple et al., 2001; Trussell et al., 2002). However, trait-mediated indirect effects can result from any type of interaction (predatory or not) which alters the interaction between two or more other species (Wootton, 1993, 2002). So a complete picture of structuring factors in communities would incorporate these 'hidden arrows' connecting seemingly disjunct species. Such hidden arrows are perhaps the basis of all emergent community effects.

Finally, while a given species must deal with numerous interactions and potentially mitigate several diverse agents of selection, they can use a variety of traits to this end. That is, they have multiple traits to mitigate their multitude of selection pressures. Again, rather than expecting singular interactions to be additive, we can expect interesting yet empirically accessible patterns to emerge. Combinations of traits can be crafted (evolutionarily) to provide integrated adaptations (i.e. trait integration, sensu DeWitt et al., 1999). For example, traits might be used in a compensatory manner, such as behavioural responses that compensate for morphological vulnerabilities. Such an instance occurs in fish-crayfish interactions. More vulnerable life stages of cray- 
fish exhibit a greater degree of behavioural response to predatory fishes (Stein and Magnuson, 1976; Stein, 1977; Keller and Moore, 1999, 2000), illustrating a compensatory relationship between size and behaviour. In theory, even complex traits such as phenotypic plasticity and bet-hedging can be used in an integrated manner (DeWitt and Langerhans, in press).

In this paper we review concepts and issues related to understanding community structure through integration of ecological and evolutionary subdisciplines. We emphasise simultaneous study of multiple traits and multiple agents of selection. These topics are illustrated with repeated reference to a model system (physid snails, sunfish and crayfish) for continuity, but we also provide other examples. Taken to the extreme, the pluralistic approach we prescribe would include study of all traits as they mediate all combinations of biotic and abiotic interactions. Failing that, as we must, the best we can do is to choose informative systems for study, and follow our naturalist's sense for the most important interactions and processes to study. This paper is meant mainly to identify types of interactions and processes that deserve particularly close scrutiny.

\section{Functional ecology and selection gradient analysis}

Integration of ecological and evolutionary parameters could prove highly useful in finding and quantifying community-structuring mechanisms. Despite this potential, no community ecological study of which we are aware has used a key technique of evolutionary biology, selection gradient analysis. Non-random factors structuring communities presumably stem from functional mechanisms, and these mechanisms are studied under the rubric of functional ecology. Functional ecology examines how traits mediate performance in relevant environments. Assuming that performance translates to fitness (Arnold, 1983), functional ecological research can simultaneously yield both ecological and evolutionary insight. Performance measures, almost by definition, equate with selection coefficients, yet functional ecological papers do not report these coefficients (Arnold, 1983). Selection coefficients describe the magnitude and direction of selection acting on phenotypes while also providing a standardised metric comparable across studies (Lande and Arnold, 1983). As we describe below, selection gradient analyses parameterise effects on both the number of organisms and the distribution of trait values among high and low performers.

Selection gradient analysis uses multiple regression to describe the relationship between relative fitness $(w)$ and standardised trait values $\left(z_{1 \ldots n}\right)$ for multiple traits (Lande and Arnold, 1983). The basic model is:

$w_{i j}=\beta_{0}+\beta_{1} z_{1}{ }^{\prime}+\beta_{2} z_{2}{ }^{\prime}+\ldots \beta_{n} z_{n}{ }^{\prime}$

Thus for each environment $(j)$, fitness variance among individuals $(i)$ is partitioned into effects attributable to each of $n$ traits. Multiple regression calculates a constant $\left(\beta_{0}\right)$ and $n$ partial regression coefficients (selection gradients, $\beta_{1 \ldots n}$ ). These coefficients (selection gradients) describe selection acting directly on focal traits $\left(z_{1 \ldots n}\right)$ by statistically controlling for selection acting only indirectly, through trait correlations. Stabilising selection can also be estimated, if warranted, by including polynomial trait values in the regression model (Lande and Arnold, 1983; Brodie et al., 1995). Finally, trait interactions can be included in the model to test for correlational selection (i.e. selection acting uniquely on trait combinations). Correlational selection is of interest because it drives evolution of some forms of trait integration (see Section 5).

Below we highlight three specific ways selection gradient analyses inform community studies:

1. Identification of true targets of selection, providing a focus for detailed functional ecology

2. Estimation of environment-specific mortalities comparable across studies

3. Identification of specific agents of mortality in complex food webs

First, because selection gradients identify fitness effects attributable strictly to individual traits, by controlling for indirect effects acting through trait correlations, they point to appropriate targets for detailed functional ecological work. For example, if one were to find a positive relationship between leg 
length and survivorship in an arboreal primate, it might be tempting to conclude that leg length mediates survival. However, a selection gradient analysis could reveal the only reason long-legged primates have high survivorship is that these individuals also tend to have long arms, which facilitate arboreal locomotion and serve as the true target of selection.

Second, a distinction between $\beta_{1 \ldots n}$ and $\beta_{0}$ should be made. A selection gradient $\left(\beta_{1 \ldots n}\right)$ is equivalent to a slope which specifies how specific trait values help mitigate the selective agent. On the other hand, the intercept $\left(\beta_{0}\right)$ reflects an elevation which is determined by average performance in a given environment. For example, if survivorship is used as fitness, benign environments (i.e. higher survivorship) will have a greater elevation than harsher environments. In this case, if there is twice the mortality in environment $\mathrm{B}$, its marginal mean (= intercept when using standardised trait values) will be half that of the benign environment.

Third, among the most important (and virtually unrecognised) aspects of selection gradients is their potential to disentangle agents of mortality in complex communities. This is because the slope of selection in a complex environment is a weighted compilation of actions due to each individual agent of selection. So if the selection gradient in a complex environment is intermediate between the single-factor environments, then all agents of selection contributed equally as sources of mortality. The degree to which the mixed-environment selection gradient deviates from intermediacy should reflect the bias in mortality where one agent of selection creates more mortality than the other. For example, imagine predator $\mathrm{A}$, when alone, exerts a selection gradient of $\beta_{\mathrm{i}, \mathrm{A}}=0.4$ on trait $i$ of its prey. Further, predator $\mathrm{B}$, when alone, exerts a selection gradient of $\beta_{\mathrm{i}, \mathrm{B}}=-0.4$ on this prey trait. If the selection gradient in the presence of both predators is zero, this suggests that selection for high trait values by predator $A$ is exactly offset by selection for low trait values by predator B. Such a result represents additive selection, where the sum of individual selection gradients equals the selection experienced when agents are simultaneously present. However if $\beta_{\mathrm{i}, \mathrm{A}+\mathrm{B}}>0$, this suggests predator $\mathrm{A}$ is eating the majority of prey (i.e. emergent selection).

Selection gradient analysis can be performed in laboratory or field settings. Field studies are perhaps most common (reviewed in Kingsolver et al., 2001), but even when conducted in the laboratory, selection gradient analysis can generate predictions about natural populations.

Box 1. Case study of functional ecology and selection gradient analysis

Freshwater snails face a variety of functionally diverse predators (Snyder, 1967; Tripet and Perrin, 1994; Reed and Janzen, 1999; DeWitt et al., 2000; Trussell and Smith, 2000). Previous field and laboratory studies found that certain alternative predators create functional trade-offs (divergent natural selection) for several snail traits (DeWitt et al., 2000). Recently we addressed these trade-offs more formally, and simultaneously, using selection gradient analysis (Langerhans and DeWitt, unpublished). We find that when functionally diverse predators consume snails, contrasting selection gradients arise for snail behaviour, morphology and life history. Oppositely-signed selection gradients (selection favouring positive standardised trait values in one environment but negative values in another) indicate divergent natural selection. Most significantly, we also found that divergent natural selection and its component selection gradients provide a foundation for understanding community effects.

First we wish to highlight the utility of selection gradients for identifying true targets of selection. In a mesocosm experiment, snails (Physa virgata) were exposed to predation in three alternative predator environments: crayfish, Procambarus clarkii, redear sunfish, Lepomis microlophus, and both predators combined. To ensure the expectation in our combined predator treatment was intermediate, predator densities used were half those in the single-predator treatments (i.e. $\mathrm{D}_{1+2}=1 / 2 \mathrm{D}_{1}+1 / 2 \mathrm{D}_{2}$; Soluk, 1993). We allowed 14 hours of predation and calculated selection coefficients for several snail traits (Table 1). 


\section{Box 1 (continued)}

Table 1 presents selection gradients (partial regression coefficients) and selection differentials for four snail traits. Selection differentials are the univariate equivalents of selection gradients (Lande and Arnold, 1983) and describe the total intensity of selection acting on a trait (direct and indirect selection). When fitness is survivorship, a selection differential is simply the difference in a standardised trait value among the survivors and the pre-selection individuals. So a coefficient of 0.2 means that an increase of one standard deviation in phenotype provides a $20 \%$ increase in fitness.

Due to trait correlations (see Section 5), the selective effect on one trait can be washed out, or even reversed, by compensatory effects in a correlated trait. Consider the shaded region of Table 1, which shows strong significant selection on covered habitat use. These effects were not evident in the selection differential analysis, probably because these snails are known to employ trait compensation between behaviour and morphology (DeWitt et al., 1999; Rundle and Brönmark, 2001). Since trait correlations are common, and because they are expected due to trait integration (see Section 5), this problem will pervade in studies focusing only on a single trait.

Second, selection gradient analysis provides environment-specific y-intercepts in addition to partial regression coefficients (selection gradients). Since this method is applied to standardised trait values, the yintercept is equivalent to the marginal mean fitness and provides a useful way to compare mortalities across environments and across studies. Additionally, fitness functions can be generated from selection gradients to illustrate effects of alternative environments on mortality and trait distributions of a focal species (Fig. 1). In our case study, fish caused a greater mortality than crayfish when single predator types were present, but mortality was greatest in the combined predator treatment. These patterns of mortality are indicated by circling $y$ intercepts $\left(\beta_{0}\right.$ 's) in Fig. 1 . The slopes of our fitness functions $\left(\beta_{1 \ldots n}\right)$ demonstrated that fish selected for round shells and covered habitat use in snails. Crayfish selected for elongate shells and avoidance of covered habitat in snails. These results, although produced in a mesocosm study, create predictions concerning natural snail populations. Oppositely signed selection gradients predict that snails in populations with only fish predators will exhibit round shells and frequent use of covered habitat, relative to those in populations with crayfish. For shell morphology, this prediction has been upheld by field surveys (DeWitt et al., 2000). The functional ecological details of shell morphology as it relates to antipredator defences is explored elsewhere (DeWitt et al., 2000; see also Krist, 2002). Here we focus on the numerical selection gradients themselves, especially in the combined predator treatment.

Using selection gradients, we were able to infer the primary predator responsible for prey mortality in the combined predator treatment. Note that for shell shape and behaviour - the two traits with opposite signs across environments - selection in the combined predator treatment was not intermediate (i.e. a non-additive effect emerged). The selection gradients were very similar to that in the crayfish-only treatment, or perhaps they are more exaggerated than in the crayfish-only environments (Table 1, Fig. 1). This strongly indicates that most or perhaps all predation in the combined predation treatment was due to crayfish. Our expectation was for equivalent predation, or possibly greater predation by fish in the combined treatments. However, the identity of the true predator is betrayed by its unique form of selection.

\section{Emergent impacts}

With the complexity of potential interactions among species, unexpected patterns may emerge in communities. One area of emergent community properties currently receiving focused attention is that of multiple-predator effects (Sih et al., 1998). A multiple-predator effect is indicated when knowledge of individual predator impacts on a prey species does not predict the observed impact when both predators are present. That is, a multiple-predator effect emerges when interactions at work within a community do not reflect the sum of individual effects (Fig. 2).

Predation is a primary structuring force in many communities (Sih et al., 1985; Kerfoot and Sih, 1987; Lima and Dill, 1990; Jackson et al., 2001). Virtually 
Table 1

Selection coefficients for four snail traits in the three predator environments

\begin{tabular}{|c|c|c|c|c|c|c|}
\hline \multirow[t]{2}{*}{ Prey Trait } & \multicolumn{3}{|c|}{ Selection Gradient ( $\left.B^{\prime}\right)$} & \multicolumn{3}{|c|}{ Selection Differential $\left(i^{\prime}\right)$} \\
\hline & $\mathrm{CC}$ & $\mathrm{FC}$ & $\mathrm{FF}$ & $\mathrm{CC}$ & $\mathrm{FC}$ & FF \\
\hline Shell size & $0.18^{\dagger}$ & $-0.18^{\mathrm{ns} / *}$ & $0.40^{*}$ & $0.18^{*}$ & $-0.14^{\mathrm{ns} /{ }^{*}}$ & $0.35^{*}$ \\
\hline Shell aspect ratio & $0.20^{\dagger}$ & $0.32^{* / *}$ & -0.18 & 0.13 & $0.30^{* / *}$ & -0.21 t \\
\hline Covered habitat & $-0.13^{*}$ & $-0.20^{* / *}$ & $0.26^{*}$ & $-0.21^{*}$ & -0.16 & 0.06 \\
\hline Near-surface habitat & 0.03 & 0.07 & 0.23 & 0.06 & 0.14 & 0.18 \\
\hline
\end{tabular}

CC: crayfish, FF: fish, FC: fish and crayfish. ${ }^{*} \mathrm{P}<0.05 .{ }^{\mathrm{ns}}$ Not significant. ${ }^{\dagger}$ Significant under heavy predation only. ${ }^{\ddagger}$ Significant if outlier is excluded. Selection in the FC treatment was tested for deviation from both zero and from the additive expectation. Data from Langerhans and DeWitt (unpublished).

all communities contain multiple predator species (Schoener, 1989; Wilbur and Fauth, 1990; Polis, 1991; Polis and Strong, 1996; Sih et al., 1998). Clearly then, insight into emergent effects of multiple predators will greatly aid our understanding of community structure; and these effects underscore the inherent complexity of species interactions.

\section{Indirect community effects and the 'trait space' view}

The role of indirect effects in structuring communities has received much theoretical and empirical attention (reviewed in Case and Bender, 1981; Kerfoot, 1987; Billick and Case, 1994; Wootton,

Box 2. Case study of emergent impacts

Since freshwater snails are prey to diverse predator types, they represent an ideal system in which to test for emergent impacts. The mesocosm experiment described in Box 1 revealed an emergent ecological impact, where snail mortality in the presence of both predators was significantly greater than expected based on additive effects of individual predators (i.e. non-additive predation; Fig. 3, left panel). A selection gradient analysis assisted in unveiling the likely mechanism producing the emergent effect. Note for example, the parallelism between the emergent predator impact and emergent selection gradients (i.e. non-additive mortality and selection gradients). To illustrate this effect graphically, we have reproduced selection gradients for covered habitat use next to the emergent ecological impact graph below (Fig. 3). Fish presence induces covered habitat use in snails (Turner, 1996). This behaviour removes snails from the visual field and physical reach of fish predators. However, crayfish are substratum dwelling predators that frequent covered habitats. So snails seeking refuge from fish crawl into the clutches of crayfish.

In the preceding example, evolutionary parameters (selection gradients) revealed a mechanistic explanation of an emergent community ecological pattern. This explanation was made possible only by simultaneously integrating the study of multiple traits and multiple agents of selection using the standardised framework of selection gradient analysis. While these results were produced in a mesocosm setting, the utility of a multiple-trait, multiple-environment approach also can be extended to field studies. For example, enclosure/exclosure experiments could manipulate the presence of particular predators and prey mortality and trait distributions could be obtained. Field studies of this type have yet to be performed in the physid snail system. 


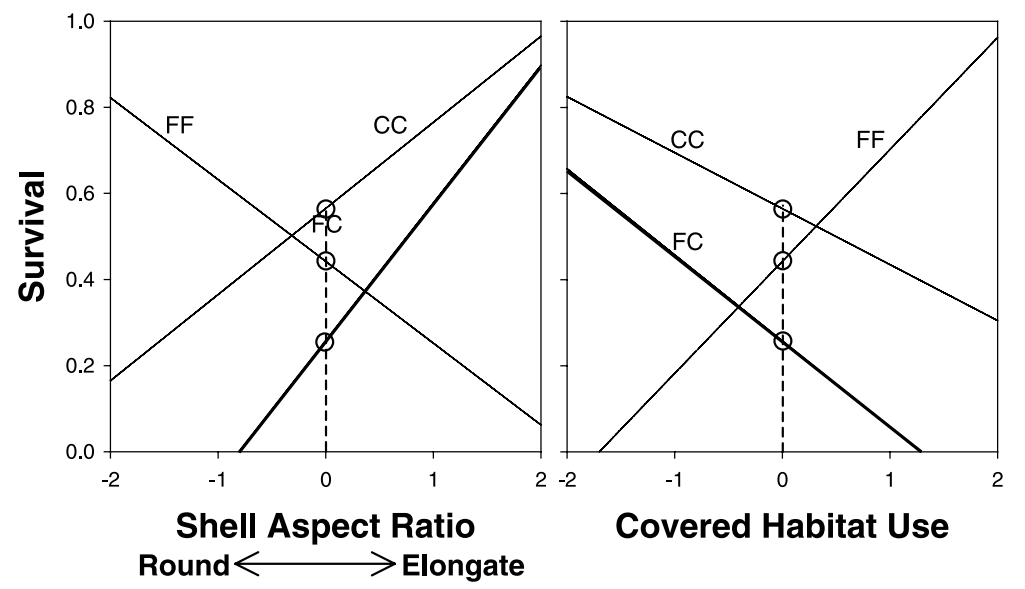

Fig. 1. Fitness functions for two snail traits in the three predator environments (CC, crayfish; FF, fish; FC, fish and crayfish). Environmentspecific y-intercepts are circled to highlight relative mortality differences across predator environments. Data from Langerhans and DeWitt (unpublished).

1994, 2002; Abrams et al., 1996; Sih et al., 1998). The two types of indirect effects are illustrated in Fig. 4. The first type, density-mediated indirect effects (also termed interaction chains, sensu Wootton, 1993), has been a major focus of community ecologists for decades. Many important ecological phenomena such as interspecific competition, trophic cascades and apparent competition result from density-mediated indirect effects (reviewed in Wootton, 1994, 2002; Pace et al., 1999). The second type, trait-mediated indirect effects (also termed

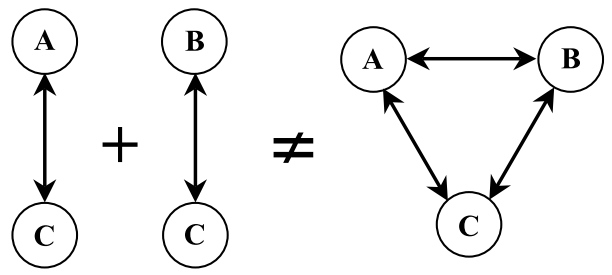

Fig. 2. Multiple-predator effect in a hypothetical community with two predators (A, B) and one prey (C). The interactions observed between predator-prey pairs when taken out of its ecological context may not be representative of the interactions within the community as a whole. So summing the effects of $\mathrm{A}$ and $\mathrm{B}$ on $\mathrm{C}$ does not necessarily equal the combined effects. interaction modifications, sensu Wootton, 1993), has only recently received critical attention (e.g. Werner, 1992; Wootton, 1993, 1994; Werner and McPeek, 1994; Abrams et al., 1996; Werner and Anholt, 1996; Schmitz, 1998; Peacor and Werner, 2001).

While we do not yet know the general importance of trait-mediated indirect effects in natural communities, many studies suggest their role rivals that of density-mediated indirect effects (e.g. Beckerman et al., 1997; Bernot and Turner, 2001; Peacor and Werner, 2001; Trussell et al., 2002). We should also stress that the labels for indirect effects (density-versus trait-mediated) can be misleading. All interactions among species, direct or indirect, are inevitably mediated by traits. In our view a community can be seen as a complex system of trait interactions. For example, any given species exhibits several types of traits (e.g. life history, morphology, behaviour), each of which can mediate interactions with other species. In addition, traits may be context-dependent (e.g. behaviour, Lima and Dill, 1990; morphology, Scheiner, 1993). Both abiotic and biotic environments can alter traits in ways that change interactions between species. The large number of possible trait interactions within even 'simple' communities creates a situation of rapidly emerging complexity. 
Yet even when indirect effects inevitably arise, one way to better understand the diversity of structuring factors is through experimentation with model systems. in the usual sense implies coordinated evolution of several traits in a focal population as phenotypes adjust to a common agent of selection (Schmalhausen, 1949). For example, imagine that populations A

Box 3. Case study of indirect community effects

Both density-and trait-mediated indirect effects occur in the snail-fish-crayfish system (e.g. Brönmark et al., 1992; Lodge et al., 1994; Turner, 1997; Turner et al., 2000; Bernot and Turner, 2001). Brönmark et al. (1992) found a density-mediated effect where pumpkinseed sunfish (Lepomis gibbosus) predation on snails indirectly increased periphytic algae biomass and dramatically modified the species composition of periphyton. Also, Bernot and Turner (2001) demonstrated a trait-mediated effect in the system where different predators caused alternative habitat shifts in snails, resulting in alternative effects on snail resources. Snails in the presence of caged pumpkinseed sunfish preferentially used covered habitats, while snails in the presence of caged crayfish (Orconectes rusticus) preferentially used near-surface habitats. These behavioural shifts resulted in large differences in periphyton standing crop: periphyton in covered habitats was $193 \%$ greater in crayfish presence and periphyton in near-surface habitats was $110 \%$ greater in sunfish presence.

Most studies on trait-mediated indirect effects to date involve behavioural changes in a focal species (e.g. Lima and Dill, 1990; Turner and Mittelbach, 1990; Huang and Sih, 1991; Werner, 1992; Schmitz et al., 1997; Ripple et al., 2001; Trussell et al., 2002). Other types of traits also influence interactions between other community members. For example, Wootton (1993) found that barnacles modify a bird-limpet interaction via shell colour. Since barnacles resemble limpets in shell colour, they reduce the ability of predatory birds to locate the limpets. Also, recent studies suggest that plastic changes in morphology and life history are important in creating trait-mediated indirect effects (Chase, 1999; Raimondi et al., 2000; Relyea, 2000; Agrawal, 2001; Langerhans and DeWitt, 2002).

Developmental phenotypic plasticity, much like behavioural flexibility, might greatly contribute to the context-dependent nature of species interactions within communities. Plasticity, environmentally contingent phenotypic expression, should enhance the likelihood that the nature of interactions among several species would change across environments. For instance, Relyea (2000) found that predatorinduced morphological plasticity in tadpoles altered competitive interactions among tadpoles. Additionally, Langerhans and DeWitt (2002) demonstrated a morphological change in snails in response to nonpredatory members of its community that enhances their vulnerability to actual predators. Knowledge of the physid snail system suggests that such interactions will translate into important cascades throughout the food web (e.g. increased periphyton levels, increased crayfish density, decreased macrophyte density).

\section{Trait integration}

The concept of trait integration is an extension of co-adaptation theory. In trait integration, however, several types of interrelationships of traits are explicitly recognised at the individual level. Co-adaptation and $\mathrm{B}$ are initially both adapted to common conditions, but then the environment changes for population B. Several traits perhaps bear on adapting to the new environment. The first trait to move will be that with the greatest product of selection and additive genetic variance. Once the first trait adjusts 


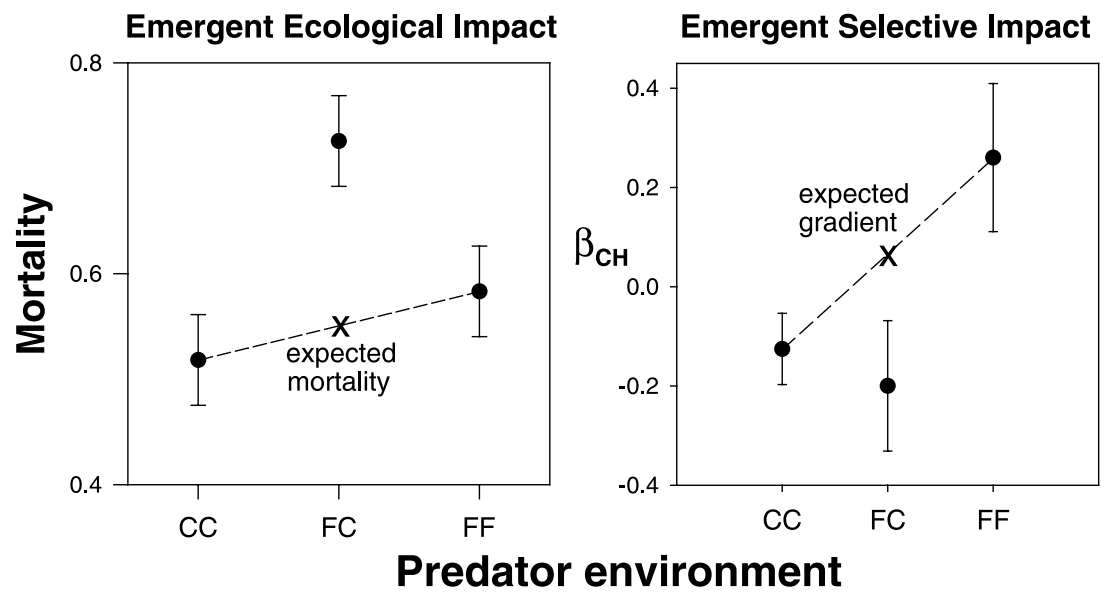

Fig. 3. Emergent ecological and selective impacts. Left panel: snail mortality in the three alternative predator environments (CC, crayfish; FF, fish; FC, fish plus crayfish). Right panel: selection gradients for covered habitat use $\left(\beta_{\mathrm{CH}}\right)$ for the three environments. Negative selection gradient values indicate that covered habitat use reduces fitness; positive values indicate the behaviour is adaptive. Additive expectations in the combined predator environment are indicated with X's in each panel. Values represent means \pm 1 SE.

to the new environment, interactions of other traits to accommodate the first change must take place to provide an integrated, or 'harmonious' overall adaptation. Thus, summing across populations, trait correlations emerge- some organisms have trait suite $\mathrm{A}$ and some have trait suite B resulting from coadaptation.

Similarly, trait integration is about ways traits are correlated, but specifically addresses how traits are correlated within populations (Table 2). The nature of these trait correlations will often be the direct result of

\section{Density-mediated indirect effect}

A

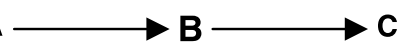

Fig. 4. The two types of indirect effects. In both cases, species A indirectly affects species C. Left, a chain of direct interactions results in an indirect effect of species $A$ on species $C$; species $A$ directly impacts B and B directly impacts C. Right, species A indirectly affects species $\mathrm{C}$ by altering the interaction between species B and C. Here, species A either alters a trait of B or C, or it alters the environmental context of the interaction between species B and C. Adapted from Wootton (1993). natural selection. For example, often two traits must be used in a complementary manner, such that the fitness conferred by a specific value of trait A depends

Table 2

Types of trait integration

\begin{tabular}{ll}
\hline Codependence: & Mechanical dependence between traits. \\
& For example, where swimming speed \\
& and tail size are correlated because \\
& one trait is strictly determined \\
& by another. \\
Complementation: & A correlation between mechanically \\
& independent traits where traits must \\
& be used in specific combinations to be \\
& effective. Here the functional \\
& significance of a given trait value \\
& depends on the value of another trait. \\
& Negative correlation between \\
& mechanically and functionally independent \\
traits as they impact fitness. & For example, morphologically vulnerable \\
prey may compensate with increased & antipredator behaviour, or vice versa. \\
Positive correlation between \\
mechanically and functionally \\
independent traits. For example, \\
a subset of individuals are \\
specialised for a given environment, \\
whereas others are specialised for \\
an alternative environment.
\end{tabular}


on the value of trait B (i.e. epistasis for fitness). In garter snakes, two morphologies exist: cryptic and striped. Cryptic coloration is most effective when used with a certain type of behaviour - jumping away from potential predators followed by strict immobilisation. Striped coloration is more adaptive when snakes behaviourally flee their predators (Brodie, 1992). This form of correlation is termed 'trait complementation' (DeWitt et al., 1999).

In other cases selection will favour individuals with a negative correlation between traits, such that selection is mediated by either trait A or B, rather than by specific combinations of the traits. For example, snails that are morphologically more vulnerable to predators perform more defensive behaviours (DeWitt et al., 1999; Rundle and Brönmark, 2001). This form of correlation is termed 'trait compensation'. Traits may also be mechanically linked ('trait codependence') such as the requirement of certain tail morphologies to enact faster escape swimming. Finally, traits may be mechanically and functionally independent, but be positively correlated in terms of their effect on fitness ('trait cospecialisation'). community ecology is required. Integration of subdisciplines (inter- and multi-disciplinary research) is becoming increasingly common (e.g. JSR 47: 3/4), and this trend is being encouraged by research funding agencies (Mervis, 1999). The recent NIOZ symposium, 'Structuring Factors in Shallow Marine Coastal Communities' from which several papers in the present issue of JSR derive, also attests to this trend (see also JSR 48:2). Our goal in the present paper is to point out several profitable avenues for a pluralistic ecology.

We discussed four major areas we believe provide beneficial routes for a more integrative community ecology. Specifically, we emphasised the need for multiple-trait and multiple-species approaches. We argued that selection gradient analysis can serve as a natural bridge between functional and community ecology. Selection gradients simultaneously reveal information about ecology ( $\beta_{0}$ 's, environment-specific performances) and evolutionary trajectories $\left(\beta_{1} \ldots \mathrm{n}\right.$, trait differences between low and high performers). We argued that emergent effects are likely to abound in natural communities (e.g. multiple-predator effects,

Box 4. Case study of trait integration

Trait compensation has been shown at two scales in the snail system: within and between species. For example, DeWitt et al. (1999) found that physid snails (P. heterostropha) behaviourally compensate for morphological vulnerabilities. Vulnerability to crayfish is largely a function of snail size-increasing size decreases vulnerability up to about $10-\mathrm{mm}$ shell length, after which there seems to be an absolute refuge from predation (Alexander and Covich, 1991). Fig. 5 shows the compensatory relationship between behaviour and morphology, with vulnerable size classes indicated by shading. Rundle and Brönmark (2001) found a similar trend across species of snails, where snail species having thinner shells (more vulnerable to predation) exhibited a greater amount of antipredator behaviour. Note that if one were to measure only a single trait, say behaviour or morphology alone, net selection would be minimised because vulnerabilities due to one trait would be offset by defence conferred by the unmeasured trait.

One of the important points to highlight here, besides the interesting adaptive nature of trait integration, is that a multiple-trait and multiple-environment perspective used in combination with selection gradient analysis provides a richer and less error-prone set of answers regarding the functional ecology of the system.

\section{General conclusions}

Since multiple processes contribute to the complexity of natural systems, a pluralistic approach to
Sih et al., 1998), reflecting the complex nature of species interactions. Such complexity results in a network of interactions, the nature and consequences of which are often dependent upon third parties (i.e. 


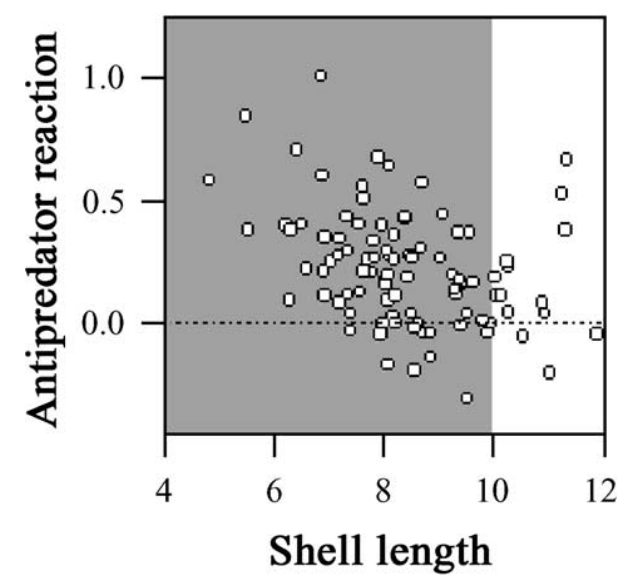

Fig. 5. Trait compensation in physid snails. Vulnerability of snails decreases with size until snails reach a size refuge at $10-\mathrm{mm}$ shell length (vulnerable sizes are indicated by shading). Small snails compensated for their morphological vulnerability with heightened antipredator behaviour (from DeWitt et al., 1999).

indirect effects, Kerfoot, 1987; Wootton, 1993; Abrams et al., 1996). Although organisms face multiple selection agents in natural communities, they also have multiple traits with which to mitigate these selection pressures. Multiple traits provide not only a battery of possible defences but also the potential for evolution of adaptive trait combinations (i.e. trait integration).

Communities often are described as complex webs of interactions (Polis and Winemiller, 1996). Here we emphasise that each interaction, direct or indirect, is likely to be trait-mediated. In fact multiple traits often will mediate given interactions. Our case studies illustrated how given predator-prey interactions were mediated by behaviour, morphology and life history traits (e.g. Table 1). Often it will be difficult to disentangle specific mechanisms that act simultaneously. Predators impact prey density through direct consumption, but also alter prey traits, either through biased consumption or by inducing phenotypic variations (phenotypic plasticity of prey). Note that both differential predation and induced responses possibly make the prey more vulnerable to other predator types (interaction modification). Or, predators may simply elevate stress in prey causing reduced growth, and only indirectly, as mediated by changing prey traits, affect prey abundance (Werner and Anholt, 1996; Boonstra et al., 1998; McPeek et al., 2001). Thus for every direct effect in a community there may be one or more important indirect effects.

So far research has focused on direct effects. Research on indirect effects is rapidly increasing (reviewed in Wootton, 2002), but most studies simply describe how a given species affects the abundance or demography of another, rather than focusing on how traits mediate interspecific interactions. Identifying specific traits that mediate an interaction provides two benefits. First, one can understand the pattern in terms of cause, so that we have a priori predictive abilities for novel systems. For example, knowing the traits mediating a given interaction we can know whether a study in the Gulf of Mexico should be generalisable to the Wadden Sea. Secondly, and perhaps more importantly, understanding how a trait mediates an interaction can serve as a starting point to deduce the possible suite of interacting traits that mediate other interactions outside of the focal interaction. That is, defining an interaction in terms of traits paves the way to understand the diversity of mechanisms generating community patterns.

Our case studies have illustrated the complexities of natural systems and the utility of a pluralistic approach. And yet the model system referenced (snailfish-crayfish) is relatively simple, comprising only three species. Understanding larger interaction webs is unquestionably daunting, but a pluralistic approach combining several subdisciplines of ecology and evolution should facilitate progress. Although such integration could take many conceivable forms, we have emphasised four topics we believe will aid discovery of mechanisms structuring natural communities.

\section{Acknowledgements}

Our work was supported by National Science Foundation grant DEB-9908528. We would also like to thank NIOZ for sponsoring the symposium 'Structuring Factors of Shallow Marine Coastal Communities,' from which this contribution derives. Theunis Piersma, Kirk Winemiller and an anonymous reviewer provided valuable comments on the manuscript. 


\section{References}

Abrams, P.A., Menge, B.A., Mittelbach, G.G., Spiller, D., Yodzis, P., 1996. The role of indirect effects in food webs. In: Polis, G., Winemiller, K. (Eds.), Food Webs: Integration of Patterns and Dynamics. Chapman \& Hall, New York, pp. 371-395.

Agrawal, A.A., 2001. Phenotypic plasticity in the interactions and evolution of species. Science 294, 321-326.

Alexander, J.E.J., Covich, A.P., 1991. Predation risk and avoidance behaviour in two freshwater snails. Biol. Bull. 180, 387-393.

Arnold, S.J., 1983. Morphology, performance and fitness. Am. Zool. 23, 347-361.

Beckerman, A.P., Uriarte, M., Schmitz, O.J., 1997. Experimental evidence for a behaviour-mediated trophic cascade in a terrestrial food chain. Proc. Natl. Acad. Sci. USA 94, 10735-10738.

Bell, G., 2001. Neutral macroecology. Science 293, 2413-2418.

Bernot, R.J., Turner, A.M., 2001. Predator identity and trait-mediated indirect effects in a littoral food web. Oecologia 129, $139-146$.

Billick, I., Case, T.J., 1994. Higher order interactions in ecological communities: what are they and how can they be detected? Ecology 75, 1529-1543.

Boonstra, R., Hik, D., Singleton, G.R., Tinnikov, A., 1998. The impact of predator-induced stress on the snowshoe hare cycle. Ecol. Monogr. 79, 371-394.

Brodie III, E.D., 1992. Correlational selection for color pattern and antipredator behaviour in the garter snake Thamnophis ordinoides. Evolution 46, 1284-1298.

Brodie III, E.D., Moore, A.J., Janzen, F.J., 1995. Visualizing and quantifying natural selection. Trends Ecol. Evol. 10, 313-318.

Brönmark, C., Klosiewski, S.P., Stein, R.A., 1992. Indirect effects of predation in a fresh-water, benthic food-chain. Ecology 73, $1662-1674$

Case, T.J., Bender, E.A., 1981. Testing for higher order interactions. Am. Nat. 118, 920-929.

Chase, J.M., 1999. To grow or to reproduce? The role of life-history plasticity in food web dynamics. Am. Nat. 154, 571-586.

Connell, J.H., 1983. On the prevalence and relative importance of interspecific competition: evidence from field experiments. Am. Nat. 122, 661-696.

DeWitt, T.J., Langerhans, R.B., in press. Integrated solutions to environmental heterogeneity: theory of multimoment reaction norms. In: DeWitt, T.J., Scheiner, S.M. (Eds.), Phenotypic Plasticity. Functional and Conceptual Approaches. Oxford University Press, New York.

DeWitt, T.J., Sih, A., Hucko, J.A., 1999. Trait compensation and cospecialization in a freshwater snail: size, shape and antipredator behaviour. Anim. Beh. 58, 397-407.

DeWitt, T.J., Robinson, B.W., Wilson, D.S., 2000. Functional diversity among predators of a freshwater snail imposes an adaptive trade-off for shell morphology. Evol. Ecol. Res. 2, $129-148$.

Goldberg, D.E., Barton, A.M., 1992. Patterns and consequences of interspecific competition in natural communities: a review of field experiments with plants. Am. Nat. 139, 771-801.

Gotelli, N.J., Graves, G.R., 1996. Null Models in Ecology. Smithsonian Institution Press, Washington DC.
Grover, J.P., 1997. Resource Competition. Chapman \& Hall, London.

Huang, C., Sih, A., 1991. Experimental studies on direct and indirect interactions in a three trophic-level system. Oecologia 85 , $530-536$.

Hubbell, S.P., 2001. The Unified Neutral Theory of Biodiversity and Biogeography. Princeton University Press, Princeton, NJ.

Jackson, D.A., Peres-Neto, P.R., Olden, J.D., 2001. What controls who is where in freshwater fish communities-the roles of biotic, abiotic, and spatial factors. Can. J. Fish. Aquat. Sci. 58, 157-170.

Keller, T.A., Moore, P.A., 1999. Effects of ontogeny and odors on behaviour: the influence of crayfish size and fish odors on crayfish movement. Mar. Freshw. Beh. Phys. 33, 35-50.

Keller, T.A., Moore, P.A., 2000. Context-specific behaviour: crayfish size influences crayfish-fish interactions. J. N. Am. Benthol. Soc. 19, 344-351.

Kerfoot, W.C., 1987. Cascading effects and indirect pathways. In: Kerfoot, W.C., Sih, A. (Eds.), Predation: Direct and Indirect Impacts on Aquatic Communities. University Press of New England, Hanover, NH, pp. 57-70.

Kerfoot, W.C., Sih, A., 1987. Predation: Direct and Indirect Impacts on Aquatic Communities. University Press of New England, Hanover, $\mathrm{NH}$.

Kingsolver, J.G., Hoekstra, H.E., Hoekstra, J.M., Berrigan, D., Vignieri, S.N., Hill, C.E., Hoang, A., Gibert, P., Beerli, P., 2001. The strength of phenotypic selection in natural populations. Am. Natural, 157.

Krist, A.C., 2002. Crayfish induce a defensive shell shape in a freshwater snail. Invert. Biol. 121, 235-242.

Lande, R., Arnold, S.J., 1983. The measurement of selection on correlated characters. Evolution 37, 1210-1226.

Langerhans, R.B., DeWitt, T.J., 2002. Plasticity constrained: overgeneralized induction cues cause maladaptive phenotypes. Evol. Ecol. Res. 4, 857-870.

Lima, S.L., Dill, L.M., 1990. Behavioural decisions made under the risk of predation: a review and prospectus. Can. J. Zool. 68, 619-640.

Lodge, D.M., Kershner, M.W., Aloi, J.E., Covich, A.P., 1994. Effects of an omnivorous crayfish (Orconectes rusticus) on a freshwater littoral food web. Ecology 75, 1265-1281.

Losos, J.B., 1990. Ecomorphology, performance capability, and scaling of West-Indian anolis lizards: an evolutionary analysis. Ecol. Monogr. 60, 369-388.

Losos, J.B., 1996. Phylogenetic perspectives on community ecology. Ecology 77, 1344-1354.

McPeek, M.A., 1996. Linking local species interactions to rates of speciation in communities. Ecology 77, 1355-1366.

McPeek, M.A., Grace, M., Richardson, J.M.L., 2001. Physiological and behavioural responses to predators shape the growth/predation risk trade-off in damselflies. Ecology 82, 1535-1545.

Mervis, J., 1999. Biocomplexity blooms in NSF's research garden. Science 286, 2068-2069.

Miller, T.E., Travis, J., 1996. The evolutionary role of indirect effects in communities. Ecology 77, 1329-1335.

Pace, M.L., Cole, J.J., Carpenter, S.R., Kitchell, J.F., 1999. Trophic cascades revealed in diverse ecosystems. Trends Ecol. Evol. 14, 483-488. 
Peacor, S.D., Werner, E.E., 2001. The contribution of trait-mediated indirect effects to the net effects of a predator. Proc. Natl. Acad. Sci. USA 98, 3904-3908.

Polis, G.A., 1991. Complex trophic interactions in deserts: an empirical critique of food-web theory. Am. Nat. 138, 123-155.

Polis, G.A., Strong, D.R., 1996. Food web complexity and community dynamics. Am. Nat. 147, 813-846.

Polis, G.A., Winemiller, K.O., 1996. Food Webs: Integration of Patterns and Dynamics. Chapman \& Hall, New York.

Raimondi, P.T., Forde, S.E., Delph, L.F., Lively, C.M., 2000. Processes structuring communities: evidence for trait-mediated indirect effects through induced polymorphisms. Oikos 91, $353-361$.

Reed, W.L., Janzen, F.J., 1999. Natural selction by avian predators on size and colour of a freshwater snail (Pomacea flagellata). Biol. J. Linn. Soc. 67, 331-342.

Relyea, R.A., 2000. Trait-mediated indirect effects in larval anurans: reversing competition with the threat of predation. Ecology $81,2278-2289$.

Ripple, W.J., Larsen, E.J., Renkin, R.A., Smith, D.W., 2001. Trophic cascades among wolves, elk and aspen on Yellowstone National Park's northern range. Biol. Cons. 102, 227-234.

Rundle, S.D., Brönmark, C., 2001. Inter- and intraspecific trait compensation of defence mechanisms in freshwater snails. Proc. R. Soc. Lond. B, Biol. Sci. 268, 1463-1468.

Scheiner, S.M., 1993. Genetics and evolution of phenotypic plasticity. Ann. Rev. Ecol. Syst. 24, 35-68.

Schmalhausen, I.I., 1949. Factors of Evolution: The Theory of Stabilizing Selection. The University of Chicago Press, Chicago.

Schmitz, O.J., 1998. Direct and indirect effects of predation and predation risk in old-field interaction webs. Am. Nat. 151, 327-342.

Schmitz, O.J., Beckerman, A.P., O’brien, K.M., 1997. Behaviourally mediated trophic cascades: effects of predation risk on food web interactions. Ecology 78, 1388-1399.

Shoener, T.W., 1983. Field experiments on interspecific competition. Am. Nat. 122, 240-285.

Schoener, T.W., 1989. Food webs from the small to the large. Ecology 70, 1559-1589.

Sih, A., Crowley, P.H., McPeek, M.A., Petranka, J., Strohmeier, K., 1985. Predation, competition, and prey communities: a review of field experiments. Ann. Rev. Ecol. Syst. 16, 269-311.

Sih, A., Englund, G., Wooster, D., 1998. Emergent impacts of multiple predators on prey. Trends Ecol. Evol. 13, 350-355.

Snyder, N.F.R., 1967. An alarm reaction of aquatic gastropods to intraspecific extract. Memoir 403 of the Cornell University Agricultural Experiment Station.

Soluk, D.A., 1993. Multiple predator effects: predicting combined functional response of stream fish and invertebrate predators. Ecology 74, 219.

Stein, R.A., 1977. Selective predation, optimal foraging, and the predator-prey interaction between fish and crayfish. Ecology 58, $1237-1253$.

Stein, R.A., Magnuson, J.J., 1976. Behavioural response of crayfish to a fish predator. Ecology 57, 751-761.

Tripet, F., Perrin, N., 1994. Size-dependent predation by Dugesia lugubris (Turbellaria) on Physa acuta (Gastropoda): experiments and model. Func. Ecol. 8, 458-463.

Trussell, G.C., Smith, L.D., 2000. Induced defenses in response to an invading crab predator: An explanation of historical and geographic phenotypic change. Proc. Natl. Acad. Sci. USA 97, 2123-2127.

Trussell, G.C., Ewanchuk, P.J., Bertness, M.D., 2002. Field evidence of trait-mediated indirect interactions in a rocky intertidal food web. Ecol. Lett. 5, 241-245.

Turner, A.M., 1996. Freshwater snails alter habitat use in response to predation. Anim. Beh. 51, 747-756.

Turner, A.M., 1997. Contrasting short-term and long-term effects of predation risk on consumer habitat use and resources. Beh. Ecol. $8,120-125$.

Turner, A.M., Mittelbach, G.G., 1990. Predator avoidance and community structure: interactions among piscivores, planktivores, and plankton. Ecology 71, 2241-2254.

Turner, A.M., Bernot, R.J., Boes, C.M., 2000. Chemical cues modify species interactions: the ecological consequences of predator avoidance by freshwater snails. Oikos $88,148-158$.

Webb, C.O., 2000. Exploring the phylogenetic structure of ecological communities: an example for rain forest trees. Am. Nat. $156,145-155$.

Werner, E.E., 1992. Individual behaviour and higher-order species interactions. Am. Nat. 140, S5-S32.

Werner, E.E., Anholt, B.R., 1996. Predator-induced behavioural indirect effects: consequences to competitive interactions in anuran larvae. Ecology 77, 157-169.

Werner, E.E., McPeek, M.A., 1994. Direct and indirect effects of predators on two anuran species along an environmental gradient. Ecology 75, 1368-1382.

Wilbur, H.M., Fauth, J.E., 1990. Experimental aquatic food webs: interactions between two predators and two prey. Am. Nat. 135, 176-204.

Winemiller, K.O., 1991. Ecomorphological diversification in lowland freshwater fish assemblages from five biotic regions. Ecol. Monogr. 61, 343-365.

Wootton, J.T., 1993. Indirect effects and habitat use in an intertidal community: interaction chains and interaction modifications. Am. Nat. 141, 71-89.

Wootton, J.T., 1994. The nature and consequences of indirect effects in ecological communities. Ann. Rev. Ecol. Syst. 25, 443-466.

Wootton, J.T., 2002. Indirect effects in complex ecosystems: recent progress and future challenges. J. Sea Res. 48, 157-172. 